\title{
Numerical Analysis of Inverted Notched Fin Array Using Natural Convection
}

\author{
Salila Ranjan Dixit ${ }^{1}$, DrTarinicharana Panda ${ }^{2}$ \\ ${ }^{I}$ (Mechanical engineering department, CMJ University, Meghalaya, India) \\ ${ }_{2}^{2}$ (Principal, OEC, BBSR, Orissa, India)
}

\begin{abstract}
Geometry and orientation plays an important role in natural convection heat transfer. For horizontal rectangular fin array a chimney flow pattern is developed due to density difference. This flow pattern creates a stagnant zone near central bottom region. That portion does not contribute much towards heat dissipation. This area is removed from fins and they became inverted notched fins. This modified geometry reduces material cost, material weight without hampering heat transfer rate. Numerical models are prepaid to investigate heat transfer characteristics in plane fins and inverted notched fins. This investigation is also extended over different types of notches and their effectiveness comparison. Fin spacing, fin height, fin length, heater input, percentage of area removed in the form of inverted notch are the parameters under consideration. This analysis is done numerically using CFD package (Fluent). It is found that the heat transfer coefficient of inverted notch fin array is 25\% to 35\% higher as compared with normal fin array. Also we found that the triangular shape notch gives better result than trapezoidal and rectangular shape notch.
\end{abstract}

Key words: Inverted Notched Fin, Chimney Flow, Natural Convection, Heat transfer coefficient enhancement.

\section{Introduction}

When available surface is found inadequate to transfer required quantity of heat with available temperature gradient, fins are used Rate of heat dissipation from a fin configuration by convection heat transfer depends on the heat transfer coefficient and the surface area of the fins. The surface area of the fins can also be increased by adding more fins to the base material in order to increase the total heat transfer from the fins. But the number of the fins should be optimized because it should be noted that adding more fins also decreases the distance between the adjacent fins. Using fins is one of the cheapest and easiest ways to dissipate unwanted heat and it has been commonly used for many engineering applications successfully. Rectangular fins are the most popular fin type because of their low production costs and high effectiveness. Configuration of all fins protruding from their bases is popular because they offer economical and trouble free solution to the problem. Natural convection heat transfer is augmented by provision of rectangular fins on horizontal or vertical surfaces in many electrical and electronic appliances. Because of reduction in surface area available for heat dissipation and low heat transfer coefficient optimization of fin geometry becomes very important in natural convection heat transfer. Now a days in electronic industries microminiaturization of electronic packages are in trend. The thermal design problem is recognized as one of the factors limiting achievement of higher packaging densities. Natural convection occurs due to temperature difference which produces the density difference. Generally in natural convection heat transfer on horizontal fin array, we observe a chimney flow pattern which creates a stagnant zone near the central bottom portion of fin channel. This stagnant zone created becomes less effective or sometimes ineffective for heat transfer, because no air stream passes over this region. To optimize the fin geometry some portion of this stagnant zone is removed in various shapes and sizes and its effect on other parameters are studied in this investigation.

\subsection{Model Analysis:}

\section{Model Analysis And Mathematical Formulation}

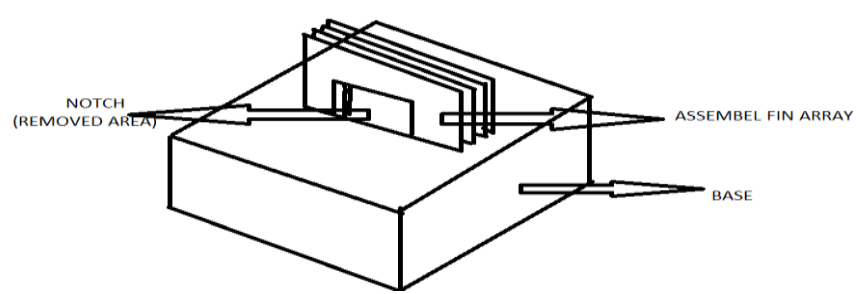

Fig 2.1: Semantic diagram of rectangular notched fin array

An array of rectangular fins is set on aluminum sheet (This model was used by Suryabansi et al. 2009) and a fixed amount of heat flux is given as in put to the base (Fig 2.1). Base is insulated from all sides except the 
on which fins are connected. Heat provided at the base is supposed to pass through the fins and the gap in between them to a surrounding that is the atmosphere. A chimney flow pattern is observed and central bottom region is untouched by this flow and become less effective. Further notches are created at the central bottom portion of the fins for examining its effect on heat transfer. The chimney flow pattern flow is shown in fig 2.2 .This chimney flow occurs due to the density difference in natural convection.

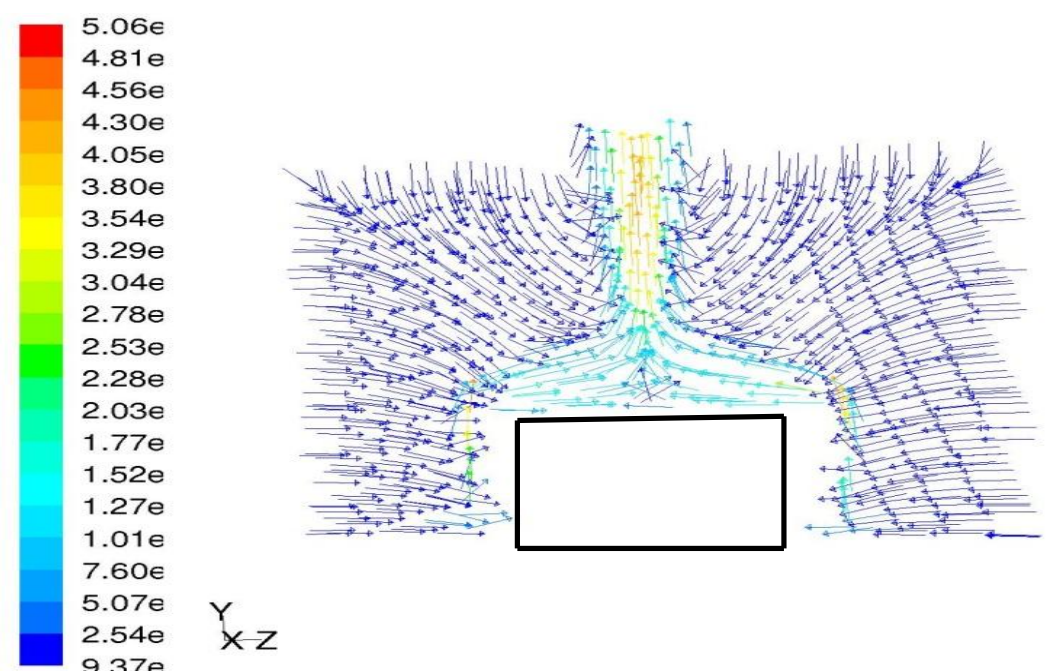

Fig 2.2 vectorialrepresentation of chimney flow

For a rectangular fin array in natural convection heat transfer.

\section{2 numerical models:}

The natural convection flow under investigation is modeled by a set of partial differential equations describing the conservation of mass, momentum, and energy in three Cartesian coordinates. The numerical model is based on control volume finite difference formulation. The set of equations are integrated over each control volume to obtain discretized linear algebraic equations, termed as finite volume equations. These equations are solved by commercial CFD package, like FLUENT. Many numbers of fins with negligible thickness is assumed. The fin surfaces with base are assumed as a source and held at uniform temperature. Laminar natural convection is the mechanism for heat transfer from the fin array. The schematic drawing of fin array under investigation with isothermal fin surface with base is shown in Fig 2.3. We are changing the percentage of notch here and also change the shape of the notch like rectangle, triangle and trapezoidal. In natural convection due to the density difference a stagnant zone is created at the central portion of fin.so we remove that area which is not affected to the flow.

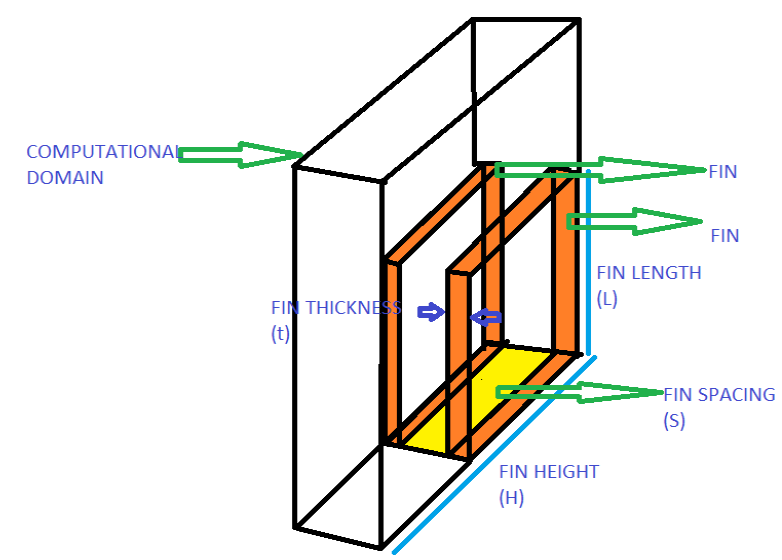

Fig 2.3: Numerical Model

The computational investigation is carried out for fin of length $\mathrm{L}$, height $\mathrm{H}$, fin spacing $\mathrm{S}$. we are taking horizontal fin array, changes space between fins accordingly the number of fin also changes given in table 2.1 .Constant heat flux given at the bottom wall shown in fig2.4. 
Table 2.1 Dimensions of rectangular fin array used for investigation.

\begin{tabular}{|llccrrr|}
\hline \multicolumn{2}{|l|}{ Length of fin array, $L(\mathrm{~mm}) 150$} & & & & \\
\hline Height, & $\mathrm{H}(\mathrm{mm})$ & 75 & & & & \\
\hline Spacing, & $S(\mathrm{~mm})$ & 4 & 5 & 6 & 9 & 7 \\
\hline No. of fins & & 15 & 13 & 12 & 9 & \\
\hline Heater input & $(\mathrm{W})$ & 50 & 100 & 150 & 200 \\
\hline
\end{tabular}

\subsection{Governing equation:}

Continuity equation

$$
\frac{\partial}{\partial x_{i}}\left(\rho U_{i}\right)=0
$$

Momentum equation

$\frac{D\left(\rho U_{i}\right)}{D t}=-\frac{\partial p}{\partial x_{i}}+\frac{\partial}{\partial x_{j}}\left[\mu\left(\frac{\partial U_{i}}{\partial x_{j}}+\frac{\partial U_{j}}{\partial x_{i}}\right)\right]-\left(\rho_{\infty}-\rho\right) \vec{g}$

$\rho$ is the fluid density anywhere in the domain and $\left(\rho_{\infty}-\rho\right)$ is the buoyancy force per unit volume. The density

$\rho$ is taken to be a function of temperature according to ideal gas law.

Energy equation

$$
\frac{D(\rho T)}{D t}=\frac{\partial}{\partial x_{i}}\left[\left(\frac{\mu}{\operatorname{Pr}}+\frac{\mu_{t}}{\operatorname{Pr}_{t}}\right) \frac{\partial T}{\partial x_{i}}\right]_{(3)}
$$

\section{4 boundary conditions:}

In natural convection flows, there is no information regarding the velocity and temperature fields before the start of calculations. Since governing equations are invariably coupled, the temperature field causes the velocity field to develop and in turn the velocity field affects the temperature field, with the promotion of convective heat transfer. The boundary conditions can be visualized both in Fig. 2.4. Constant heat flux given at the bottom wall of domain .pressure outlet boundary condition is given at top and one side of the domain.one side of the fin is given as length symmetry boundary condition. The rest two side of fin is given as width symmetry boundary condition. The lower portion of the fin is given as velocity inlet. We are taking symmetry boundary condition like width symmetry, length symmetry because it is easy doing analysis.

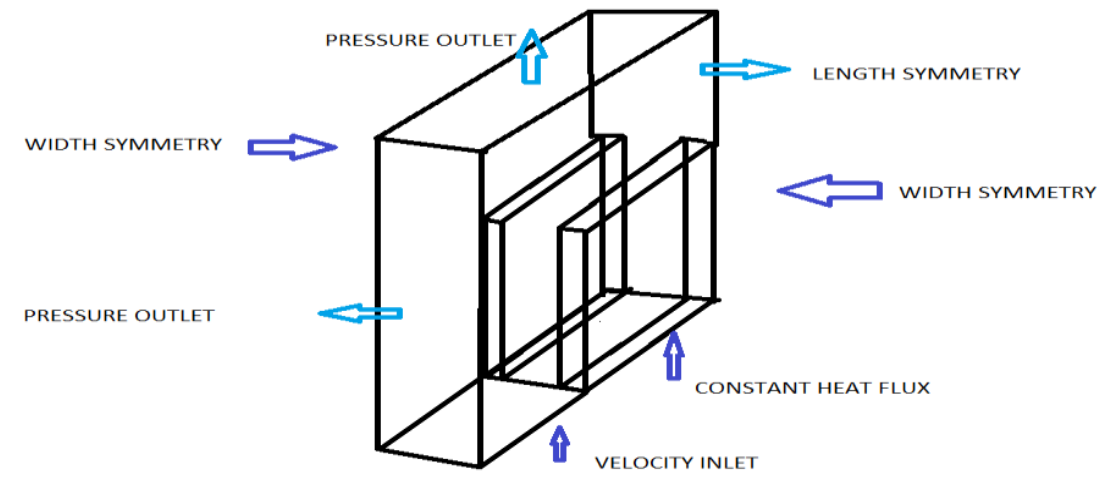

Fig. 2.4 Schematic diagram of computational domain and boundary

Velocity inlet boundary conditions are used to define the flow velocity, along with all relevant scalar properties of the flow, at flow inlets. In this case, the total (or stagnation) pressure is not fixed but will rise (in response to the computed static pressure) to whatever value is necessary to provide the prescribed velocity distribution. This boundary condition is equally applicable to incompressible and compressible flows. Pressure outlet boundary conditions require the specification of a static (gauge) pressure at the outlet boundary. The value of the specified static pressure is used only while the flow is subsonic. Should the flow become locally 
supersonic, the specified pressure will no longer be used; pressure will be extrapolated from the flow in the interior. All other flow quantities are extrapolated from the interior. A set of "backflow" conditions is also specified should the flow reverse directionat the pressure outlet boundary during the solution process. Convergence difficulties will be minimized if you specify realistic values for the backflow quantities. It is known now, beyond any doubt, that a moving fluid in contact with a solid body will not have any velocity relative to the body at the contact surface. This condition of not slipping over a solid surface has to be satisfied by a moving fluid. This is known as the no-slip conditionSymmetryboundary conditions are used when the physical geometry of interest, and the expected pattern of the flow/thermal solution, has mirror symmetry. They can also be used to model zero-shear slip walls in viscous flows. This section describes the treatment of the flow at symmetry planes and provides examples of the use of symmetry. You do not define any boundary conditions at symmetry boundaries, but you must take care to correctly define your symmetry boundary locations. Symmetry boundaries are used to reduce the extent of your computational model to a symmetric subsection of the overall physical system.

\section{5 .non dimensional parameter}

We are calculating some non-dimensional parameter like Nusselt Number, grashoff number, and Rayleigh number.

$\mathrm{Nu}=\frac{\mathrm{hL}}{\mathrm{k}}$ (Nusselt Number)

$\mathrm{Gr}=\frac{\mathrm{g} \beta \Delta \mathrm{TL}^{3}}{v^{2}}$ (Grashoffs Number)

$\mathrm{Ra}=\mathrm{Gr} * \mathrm{Pr}$

$\mathrm{Ra}=\frac{\mathrm{g} \beta \Delta \mathrm{TL}^{3}}{v^{2}} \times \frac{\mu C \mathrm{p}}{K}$

We are calculating Nusselt number because if Nusselt number is high then that means convection is more and it is directly propertional to the heat transfer coefficient and it also gives the heat transfer coefficient in non-dimensional form. We are calculating Grashoffs Number because all variable of Grashoffs number is depend upon temperature of fluid .if we found that at everywhere Grashoffs number is equal then the heat transfer rate does not change when we remove the area. We are calculating Rayleigh number because it is a function of Prandtl number and Grashoffs number. If the Rayleigh number is greater than the multiplication is power nine then the flow is turbulent and if the multiplication is power six then the flow is turbulent.

\subsection{Numerical Solution Procedure:}

Three-dimensional equations of mass, momentum and energy control volume. These equations were discretized over the control volume using the finite volume technique to yield a set of algebraic equations which could be solved by the algebraic multi grid solver of Fluent 13.1in an iterative manner by imposing the above boundary conditions. A first order upwind scheme (for convective variables) was considered for the momentum as well as for the energy equations. After a first-hand converged solution could be obtained, the scheme was changed over to second order upwind so as to get a little better accuracy (heat transfer coefficient was changed by only $0.5 \%$ ). A detail description of the types and number of grids used and the effect of domain size have been discussed separately in the results and discussions. The SIMPLE algorithm with a PRESTO (Pressure Staggering Option) scheme for the pressure velocity coupling was used for the pressure correction equation. Under relaxation factors of 0.3 for pressure, 0.7 for momentum and 1 for temperature were used for the convergence of all the variables. Simple tetrahedral cells were used for the entire computational domain due to the complexity of the geometry. Convergence of the discretized equations were said to have been achieved when the whole field residual for all the variables fell below $10^{-3}$ for $\mathrm{u}, \mathrm{v}, \mathrm{w}$, and $\mathrm{p}$ whereas for energy the residual level was kept at $10^{-6}$.Three-dimensional equations of mass, momentum and energy have been integrated over the control volume and the subsequent equations have been discretized overthe control volume using the finite volume technique to yield a set of algebraic equations which could be solved by the algebraic multi grid solver of Fluent 12.1 in an iterative manner by imposing proper boundary conditions. First order upwind scheme (for convective variables) was considered for momentum as well as for the turbulent discretized equations. After a first-hand converged solution could be obtained the scheme was changed over to second order upwind so as to get little better accuracy. SIMPLE algorithm has been used for the pressure-velocity coupling for the pressure correction equation and the cell face values of pressure could be obtained from simple arithmetic averaging of centroid values. However, it is to be noted that if density becomes a function of temperature then the SIMPLE algorithm for pressure velocity coupling will not work properly because the pressure variation from cell to cell will not be smooth due to the presence of source term in the momentum equation. So in order to obtain the cell face pressure new pressure interpolation technique is needed which is available through body force weighted or 
PRESTO (Pressure Staggered Option) scheme in Fluent. The body-force-weighted scheme computes the face pressure by assuming that the normal gradient of the difference between pressure and body forces is constant. This works well if the body forces are known a priori in the momentum equations (e.g., buoyancy and axisymmetric swirl calculations). The PRESTO (Pressure Staggering Option) scheme uses the discrete continuity balance for a "staggered" control volume about the face to compute the "staggered" (i.e., face) pressure. This procedure is similar in spirit to the staggered-grid schemes used with structured meshes. Note that for triangular, tetrahedral, hybrid, and polyhedral meshes, comparable accuracy is obtained using a similar algorithm. The PRESTO scheme for pressure interpolation is available for all meshes in Fluent. Under relaxation factors $(0.3$ for pressure, 0.7 for momentum and1for energy) were used for the convergence of all the variables. Fine grids were used at the nozzle entrance as well as for louvers opening area to have better accuracy. The field variables for all type of cells are stored at the cell center but to get cell face values, interpolation is needed from values stored at the centroids. For triangular or tetrahedral cells a node based solution procedure is adopted which evaluates the cell face values from centroid values by making some weighted mean average around the cell center and the nodes. This procedure normally makes a smooth computation of the face values (for fluxes) and helps in better convergence. Convergence of the discretized equations were said to have been achieved when the whole field residual for all the variables fell below $10^{-3}$.

\section{Results and Discussions}

\subsection{Grid independent test and validation with other results:}

We have started our numerical investigation with a rectangular fin array with fin spacing $4 \mathrm{~mm}$ and 12 numbers of fins. Subsequently it is extended to different spacing and numbers. This fin array is placed in a domain. The dimensions of the fin are length $150 \mathrm{~mm}$. height $75 \mathrm{~mm}$ and thickness $3 \mathrm{~mm}$. Fin materials is aluminum. As mentioned above in our numerical modeling we have taken two half fins(length $75 \mathrm{~mm}$ and thickness $1.5 \mathrm{~mm}$ ) for simulation applying symmetry boundary condition and accordingly dimensions of domain is taken (length $150 \mathrm{~mm}$,height $225 \mathrm{~mm}$ ).

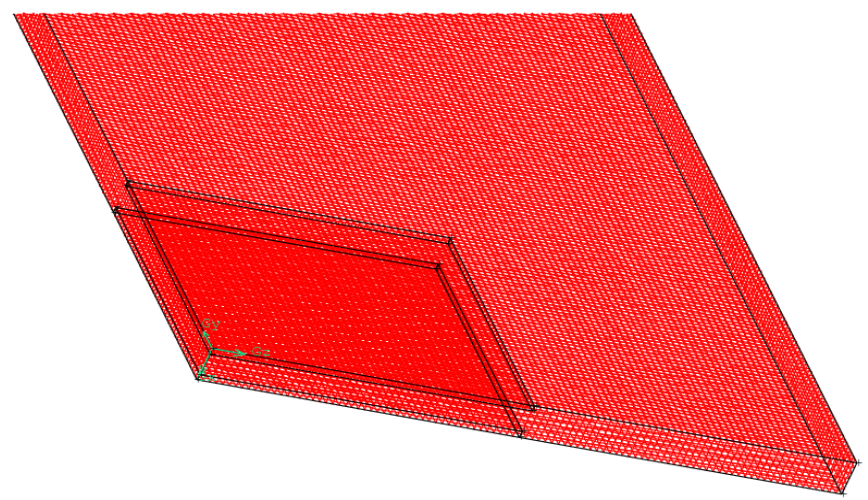

Fig 3.1:A general arrangement of meshes

Map mesh is taken here. First we have taken mesh spacing $0.003 \mathrm{~m}$ and compare the simulation result with existing experimental results. It is observed that it differs from the experimental result. Then we reduce the mesh size to $0.002 \mathrm{~m}$ and find that the resulting is approaching towards existing results. Then we further reduce the size to 0.0015 and then to 0.001 .we find the result is still approaching towards perfection and there is noticeable difference between the results of mesh size $0.0015 \mathrm{~m}$ and $0.001 \mathrm{~m}$. We also notice a remarkable change in computation time is up to 6 hours in mesh size $0.001 \mathrm{~m}$. So we have tried to match our result with existing experimental result of Suryabansi et al. (2009).We find our result match well with these experimental results. So for further all simulations we have taken this mesh size $(0.0015 \mathrm{~m})$. 


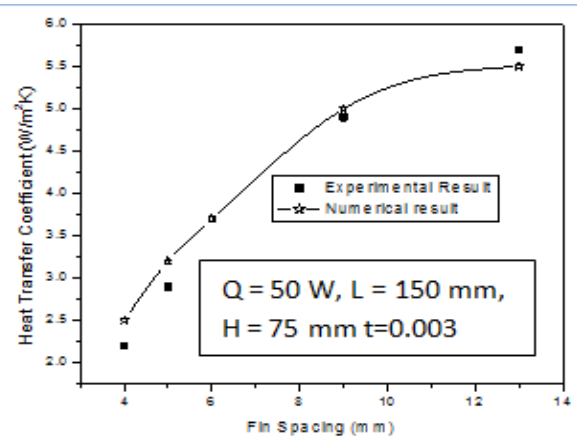

Fig. 3.1 Heat transfer coefficient as a function of fin spacing (50W)

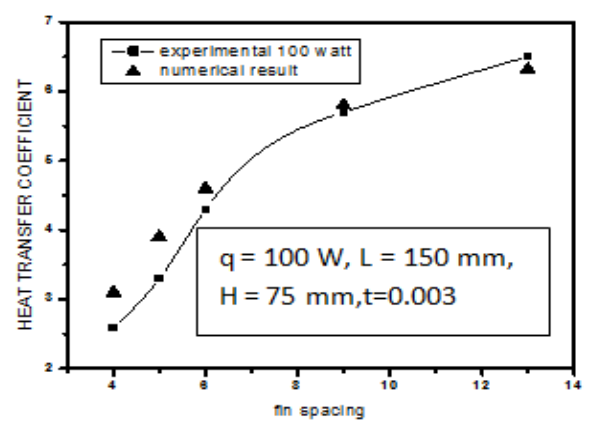

Fig. 3.2 Heat transfer coefficient as a function of fin spacing $(100 \mathrm{~W})$

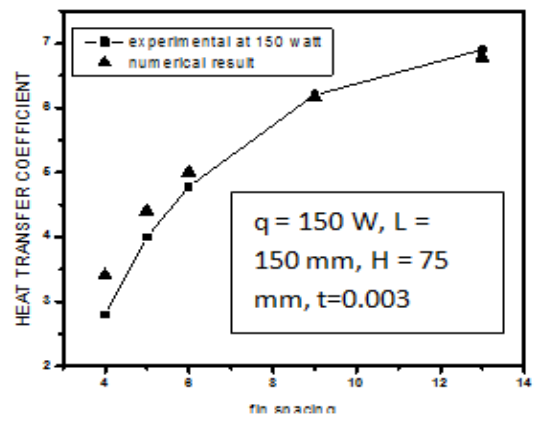

Fig. 3.3 Heat transfer coefficient as a function of fin spacing (150W)

Heat transfer coefficient is plotted as a function of fin spacing (fig ). Average heat transfer coefficient is calculated from average temperature difference between fin array and atmosphere. We have calculated heat transfer coefficient (h) for different fin spacing (s) like $4 \mathrm{~mm}, 5 \mathrm{~mm}, 6 \mathrm{~mm}, 9 \mathrm{~mm} \& 13 \mathrm{~mm}$ and different heater inputs (q) such as 50W, 100W \& 150W. Heater inputs are given in the form of constant heat flux. We have compared our result with experimental result of suryabansi et al (2009). Above figures shows the comparisons of heat transfer coefficients at different spacing between numerical results and experimental results. They show a good agreement with experimental results (with around $\pm 5 \%$ error).

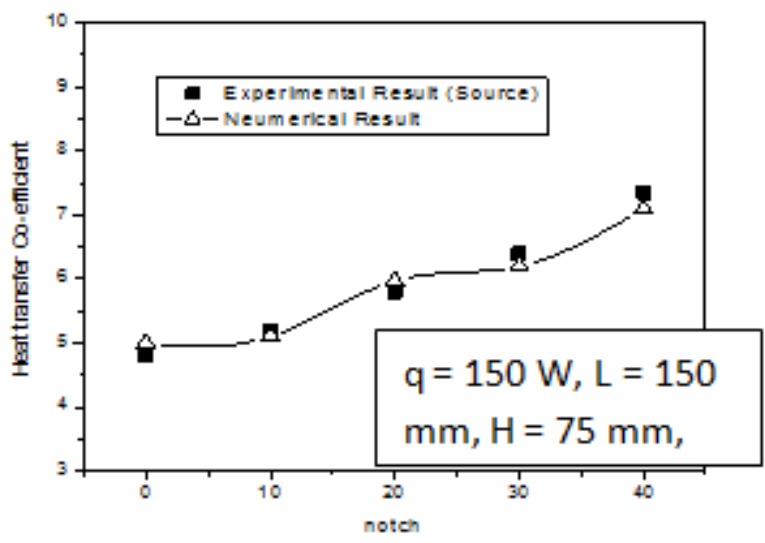

Fig.3.4 Heat transfer coefficient as a function of percentage of area removed

Heat transfer coefficient is plotted as a function of percentage of area removed (fig 3.4). Average heat transfer coefficient is calculated from average temperature difference between fin array and atmosphere. We have calculated heat transfer coefficient $(\mathrm{h})$ for different notch like (10\% removal area of fin,20\% removal of area of fin, $30 \%$ area removal of fin and $40 \%$ area removal of fin) with constant heater inputs (q) as $150 \mathrm{~W}$ as constant heat flux. We have compared our numerical result with existing experimental result of suryabansi et al (2009). They show a good agreement with each other. 


\subsection{Effect of Fin Spacing On Nusselt Number with Constant Heat Flux for Plain Fins}

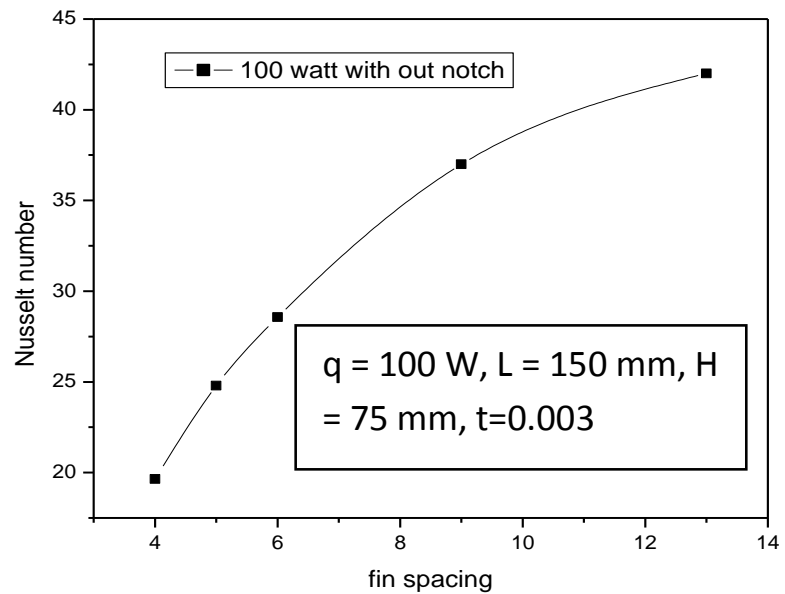

Fig 3.5 Nusselt number is a function of fin spacing

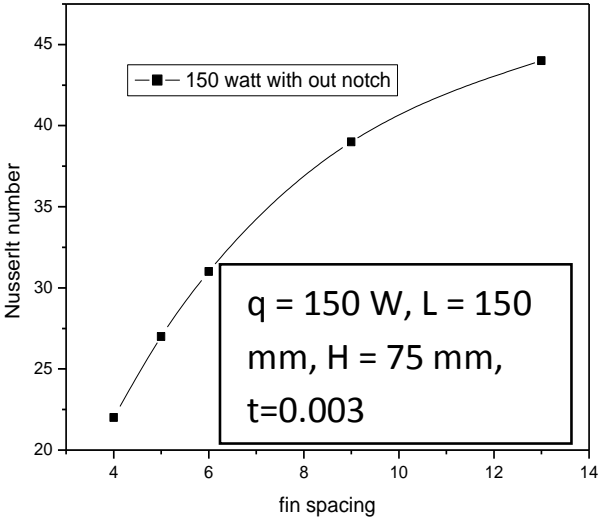

Fig 3.6 Nusselt number is a function of fin spacing

Variation of Nusselt Number with respect to fin spacing is plotted in fig no $3.5 \& 3.6$ for constant heat flux of $100 \mathrm{~W} \& 150 \mathrm{~W}$. Dimensions of fin are taken as length (L) $150 \mathrm{~mm}$, height $(\mathrm{H}) 75 \mathrm{~mm}$, and thickness $(\mathrm{T}) 3 \mathrm{~mm}$. We found that with increase in fin spacing from $4 \mathrm{~mm}$ to $13 \mathrm{~mm}$ Nusselt Number increases. But rate of increase of Nusselt Number decreases with increase in fin spacing which gives a parabolic curve. As Nusselt Number is directly proportional to heat transfer coefficient and other parameters are almost constant so it gives a curve of same nature as heat transfer coefficient, discussed earlier.

\subsection{Effect of Notch on Nusselt Number}

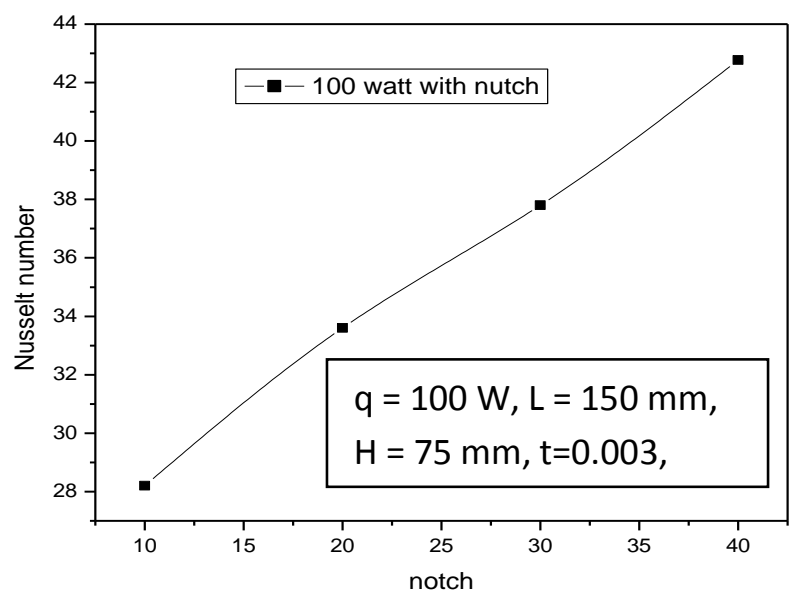

Fig 3.7 Nusselt number is a function of percentage of area removed

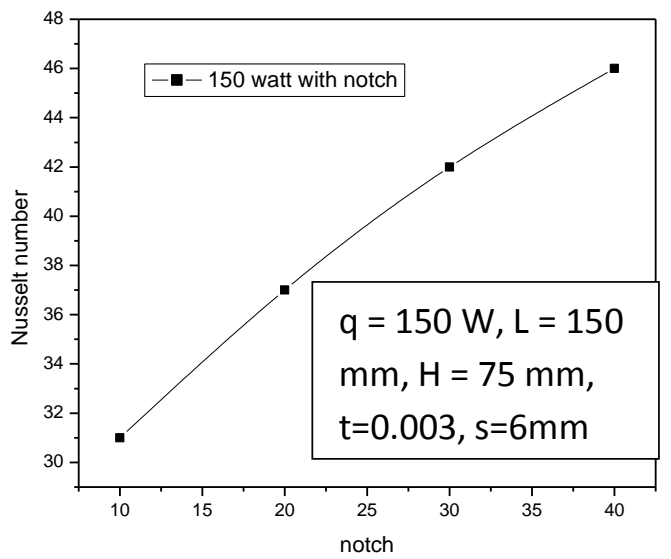

Fig 3.8. Nusselt number is a function of percentage of area removed

Rate of increase of heat transfer coefficient is sharper in case of notched fins than plain fins as discussed earlier. The major cause is removal of area without hampering heat transfer rate. In fig no 3.7 variation of Nusselt Number is plotted against percentage of area removed. And we find the Nusselt Number increases with increase in notch size. This increment is very sharp and the plot is almost a straight line unlike for plain fins. Here we have investigated on same fin structure with rectangular notches of different size starting from $10 \%$ of fin area to $40 \%$. We have tried to remove the unaffected zone by chimney flow. Here we find that if we remove the unaffected zone i.e. up to $40 \%$ of the main fin area. We find Nusselt number is increasing which implies increase in heat transfer coefficient but after that heat transfer rate is hampered. It shows that we can save up to $40 \%$ material of fins making the equipment more light and cheap. 


\subsection{Effect of Notch on Grashoffs Number}

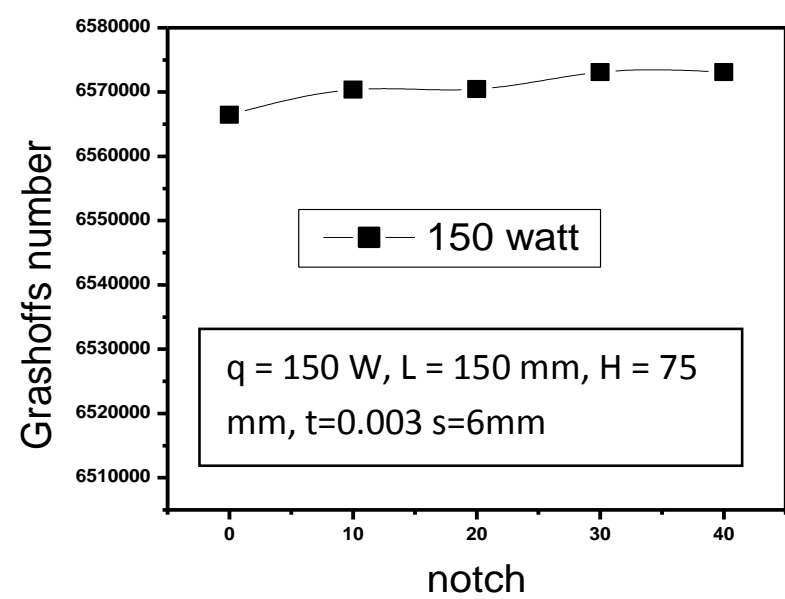

Fig 3.9.Grashoff number is a function of percentage of area removed

For optimization purpose we are removing the central bottom portion of the rectangular fin but it always carries a greater importance to maintain the original heat transfer rate that we are getting with plane fins. From fig 3.9 it is evident that Grashoffs number is almost constant with no notch condition to $40 \%$ notched. This implies the fluid temperature near to fin array remains constant even we removed the central bottom portion of the fin up to a certain extent. The constancy of fluid temperature shows that there is no appreciable change in fin temperature. From the above analysis we can conclude that as the fin temperature is not changing, the rate of heat transfer remains almost constant from no notch to $40 \%$ notch condition.

\subsection{Effect of Rayleigh Number on Nusselt Number}

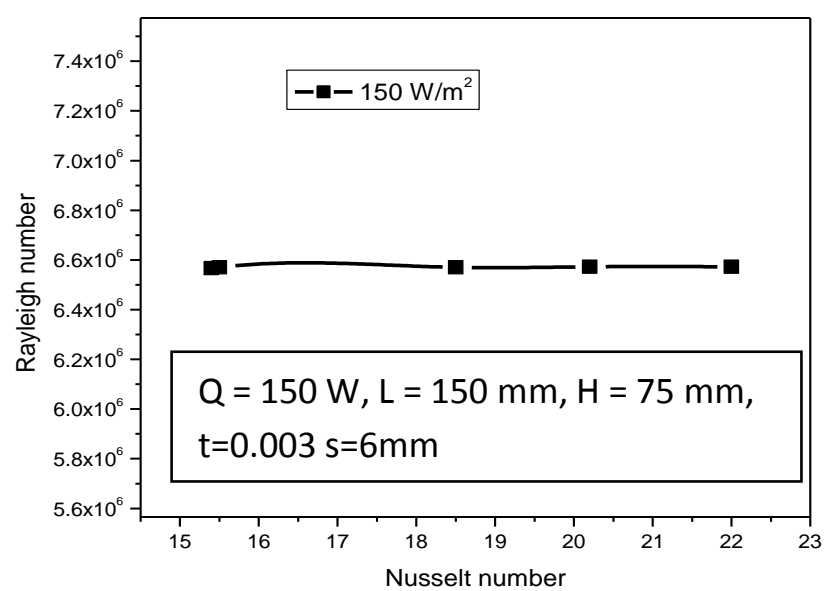

Fig 3.10. Rayleigh number is a function of Nusselt number

Density plays a major role in natural convection heat transfer. The value of viscosity varies directly with density. Viscosity determines the flow nature. It natural convection heat transfers the flow becomes turbulent at certain Rayleigh Number range $(\mathrm{Ra}=\mathrm{Gr} \times \mathrm{Pr})$ i.e. $10^{8}-10^{9}$. In our investigation we find the value of Ra lies in the range of $10^{6}$. This conforms the flow set up due to density difference is in laminar zone. The fig3.10 shows the variation of Ra with respect to different Nusselt Number. As Nusselt Number is directly proportional to heat transfer co-efficient and heat transfer co-efficient to heat supplied so in our range of investigation we find a laminar chimney flow pattern is seen in all different notches. 


\subsection{Effect of Notch on Heat Transfer Coefficient for Constant Heat Flux with Different Shape of Notch}

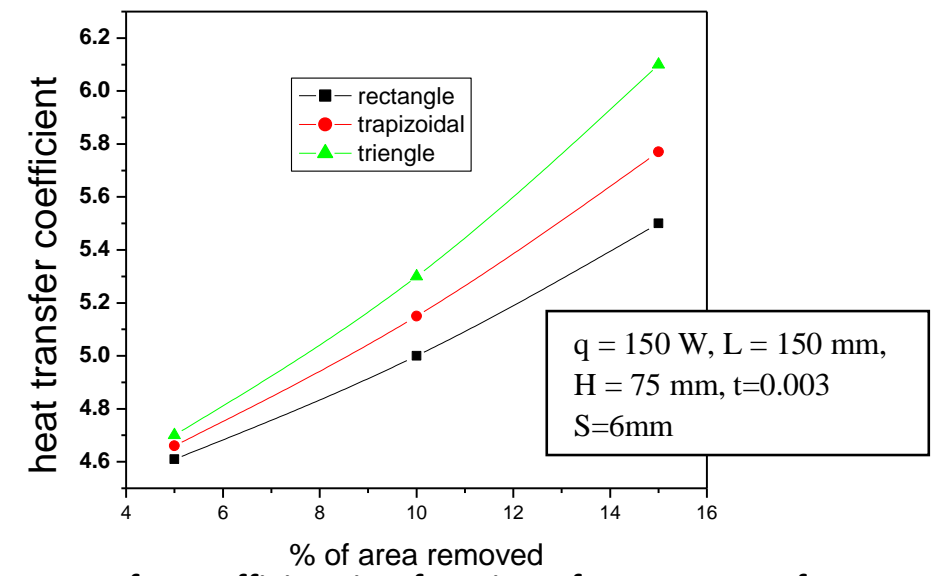

Fig 3.11. Heat transfer coefficient is a function of percentage of area removed

From the previous investigation we found that heat transfer co-efficient in case of notched fin is more than plane fins. Here we have tried to optimize the notch to get better heat transfer co-efficient with same effort. So we studied the effect of different notch shape of same size on heat transfer co-efficient. We have analyzed two more types of notches (triangular \& trapezoidal) along with rectangular notch. We compared heat transfer co-efficient of different notches at different sizes. It is observed for same conditions triangular notch give better heat transfer coefficient followed by trapezoidal and rectangular notch respectively due to more effective removal of fin area in stagnant zone. So for better optimization of fin geometry we can switch over to the above types of notches with certain limitations (Limitation like triangular and trapezoidal notch can't be made more than $20 \%$ of main fin area).

\section{Conclusions}

Fin geometry and orientation plays a vital role in natural convection heat transfer. Our investigation deals with natural convection heat transfer on rectangular fin array. Here we have tried to optimize a rectangular fin array on the basis of cost and usefulness. We have optimized above fin array based on natural convection heat transfer numerically using CFD package fluent with laminar model. Our model has been validated with existing experimental result of Suryavansi et al. we have measured heat transfer coefficients and other nondimensional parameters as a function of heat transfer coefficient. The investigation is based on rectangular fin array and the results obtained are the function of notch size and shape. The following conclusion can be derived from the present investigation: Nusselt number is increasing if we increase the fin spacing at constant heat flux for plane fins. Because Resistance to the flow due to density difference, decreases for increase in fin spacing. Nusselt Number increases with increase in notch size $10 \%$ to $40 \%$ for constant heat flux. Because the fin area decreases retaining constant heat transfer rate.TheGrashoffs Number is almost remaining same with no notch condition to $40 \%$ notch condition. This implies the fluid temperature is not changing with the change of notch size. That means the fin temperature does not change, which refers to constant Heat transfer rate. Rayleigh Number remains almost constant with increase in Nusselt Number for constant heat flux. Which gives the value of Rayleigh number gives in the order $10^{6}$. This shows the flow setup due to variation in density is laminar. The heat transfer coefficient is maximum in case of triangular (In the limit up to 20\%) shape notch followed by trapezoidal (In the limit up to 20\%) and Rectangular shaped notch (In the limit up to 20\%) at constant heat flux due to effective removal of stagnant zone fin area.

\section{Reference}

[1]. Starner K. E, and McManus H. N, 1963, "An Experimental Investigation of Free Convection Heat Transfer From Rectangular Fin Arrays," Trans. ASME,Ser. C: J. Heat Transfer, 85, pp. 273-278.

[2]. Sane N. K, and Sukhatme S. P, 1974, "Natural Convection Heat Transfer from Short Horizontal Rectangular Fin Arrays," Fifth International Heat Transfer Conference, Tokyo, Sept.

[3]. Sane N. K, Kittur M. B, and Magdum J. D., 1995, "Natural Convection Heat Transfer From Horizontal Rectangular Fin Arrays With a Rectangular Notch at the Center," Seventh ISHMT Conference, Suratkal.

[4]. Sane, N. K, Tikekar, A. N, and Morankar, K. P., 2000, "Natural Convection Heat Transfer From Vertical Arrays With Rectangular Notched Fins," ME thesis, Shivaji University, Kolhapur, India

[5]. Baskaya, S, Sivrioglu, M, and Ozek, M, 2000, "Parametric Study of Natural Convection Heat Transfer from Horizontal Rectangular Fin Arrays,” Int. J. Therm. Sci., 39, pp. 797-805.

[6]. Jones, C. D, and Smith, L. F, 1970, "Optimum Arrangement of Rectangular Fins on Horizontal Surface for Free Convection Heat Transfer," ASME Paper No. 69-HT-44. 
[7]. Harahap, F, and McManus, H. N, 1967, "Natural Convection Heat Transfer From Horizontal Rectangular Fin Arrays," Trans. ASME, Ser. C: J. Heat Transfer, 89, pp. 32-38.

[8]. Sanjeev D. Suryawanshi,Narayan K. SaneNatural Convection Heat Transfer From Horizontal Rectangular Inverted Notched Fin Arrays.

[10]. A similar experimental study was conducted by Welling .1965 Bridge on rectangular vertical fins. They reported optimum values of the ratio of fin height to spacing. In the previous two studies the fin length was constant. International journal of heat and mass transfer.

[11]. Rao V.D, Naidu S.V, Rao B.G, Sharma K.V, Heat transfer from a horizontal fin array by natural convection and radiation, International journal of heat and mass transfer, 2006,Vol. 49, pg. 3379-3391

[12]. Yüncü H. and Anbar G, "An Experimental Investigation on Performance of Rectangular Fins on a Horizontal Base in Free Convection Heat Transfer”, Heat and Mass Transfer 33, 507-514, (1998).

[13]. Guglielminiin 1986 he investigated that the heat transfers by natural convection and radiation between an isothermal vertical surface, having a staggered array of discrete vertical plates of finite thickness, and the surroundings. International journal of heat and mass transfer

[14]. Vollaro A.D.L, Grignaffini S, Gugliermetti F, "Optimum Design of Vertical Rectangular Fin Arrays", International Journal of Thermal Sciences 38, 525-259, (1999).

[15]. Leung C.W, Prober S.D. and Shilston M.J. "Heat Exchanger: Optimal Separation for Vertical Rectangular Fins Protruding from a Vertical Rectangular Base”, Applied Energy, 77-85, (1985).

[16]. Kundu B, Das P.K, "Performance and Optimum Design Analysis of Convective Fin Arrays Attached to Flat and Curved Primary Surfaces", International Journal of Refrigeration, 1-14, (2008). 92

[17] Güvenc A, "An Experimental Investigation on Performance of Rectangular Fins on a Vertical Base in Free Convection Heat Transfer”, M.S. Thesis in Mechanical Engineering, Middle East Technical University, Ankara (1999).

[18]. Orhan Aydin.2009 He takes natural convection of air in a two dimensional rectangular enclosure with localized heating from bellow and symmetrical cooling from the inside has been numerically investigated. International journal of mass transfer. 